International Journal of Pure and Applied Mathematics

Volume 88 No. 1 2013, 1-13

ISSN: 1311-8080 (printed version); ISSN: 1314-3395 (on-line version)

url: http://www.ijpam.eu

doi: http://dx.doi.org/10.12732/ijpam.v88i1.1

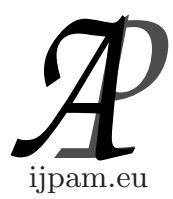

\title{
A GENERALIZED SCHEME FOR THE NUMERICAL SOLUTION OF INITIAL VALUE PROBLEMS IN ORDINARY DIFFERENTIAL EQUATIONS BY THE RECURSIVE FORMULATION OF TAU METHOD
}

\author{
K. Issa $^{1} \S$, R.B. Adeniyi ${ }^{2}$ \\ ${ }^{1}$ Department of Mathematics \\ Kwara State University Malete \\ Ilorin, NIGERIA \\ ${ }^{2}$ Department of Mathematics \\ University of Ilorin \\ Ilorin, NIGERIA
}

\begin{abstract}
The generalization of the recursive form of the tau method for both overdetermined and non-overdetermined ordinary differential equations of the initial value type is the main thrust of the work reported here. This will facilitate an automation of this variant of the method and consequently an efficient utilization of the technique.

Results from the numerical experiment confirm the validity and effectiveness of the derived scheme.
\end{abstract}

AMS Subject Classification: $34 \mathrm{~K} 28$

Key Words: Lanczos Tau method, Chebyshev polynomials, initial value problems, Lanczos-Ortiz canonical polynomial, ordinary differential equations

\section{Introduction}

The tau method of Lanczos, first proposed 1938 to solves the linear ordinary

Received: May 3, 2013

(C) 2013 Academic Publications, Ltd.

${ }^{\S}$ Correspondence author url: www.acadpubl.eu 
differential equations

$$
\begin{gathered}
L y(x) \equiv \sum_{r=0}^{m}\left(\sum_{k=0}^{N_{r}} P_{r k} x^{k}\right) y^{r}(x)=\sum_{r=0}^{\sigma} f_{r} x^{r}, \quad a \leq x \leq b, \\
L^{*} y\left(x_{r k}\right) \equiv \sum_{r=0}^{m-1} a_{r k} y^{(r)}\left(x_{r k}\right)=\alpha_{k}, \quad k=1(1) m,
\end{gathered}
$$

by seeking an approximant

$$
y_{n}(x)=\sum_{r=0}^{n} a_{r} x^{r}, \quad r<+\infty,
$$

of $y(x)$ which is the exact solution of the corresponding perturbed system

$$
\begin{gathered}
L y_{n}(x)=\sum_{r=0}^{\sigma} f_{r} x^{r}+H_{n}(x) \\
L^{*} y_{n}\left(x_{r k}\right)=\alpha_{k}, \quad k=1(1) m
\end{gathered}
$$

(see [1]-[6], [8]). In (1.1) $L$ is the differential operator, $y^{r}(x)$ is the derivative of order $\mathrm{r}$ of $y(x), P_{r k}(x), \sigma, \alpha_{k}, x_{r k}$, a and $\mathrm{b}$ are real constants and

$$
H_{n}(x)=\sum_{i=0}^{m+s-1} \tau_{i+1} T_{n-m+i+1}(x)=\sum_{i=0}^{m+s-1} \tau_{i+1} \sum_{r=0}^{n-m+i+1} C_{r}^{(n-m+i+1)} x^{r}
$$

is the perturbation term. The parameters $\tau_{i}, i=1(1)(m+s)$ in $(1.3)$ are to be determined.

$$
T_{n}(x) \equiv \sum_{r=0}^{n} C_{r}^{(n)} x^{r}=\operatorname{Cos}\left[n \operatorname{Cos}^{-1}\left(\frac{2 x-a-b}{b-a}\right)\right]
$$

is the chebyshev polynomial valid in the interval $[a, b]$. The number s, called the overdetermination number of (1.1), is given by

$$
s=\max \left\{N_{r}-r>0 \mid 0 \leq r \leq m\right\}
$$

In 1956 (see [11]), Lanczos introduced the use of canonical polynomials $\left\{Q_{r}(x)\right\}$, $r=0,1, \ldots$ into the tau method. The difficulty involved in their construction was removed by Ortiz in 1969 (see [13]), when he reported a recursive generation of the polynomials, $Q_{r}(x)$ defined by

$$
L Q_{r}(x)=x^{r}
$$




\section{Generalization of Lanczos-Ortiz Canonical Polynomials}

For the purpose of generalizing the tau approximant of the solution of $y(x)$ to equation(1.1), we shall briefly review in this section the work of Yisa (2012), see $[14]$.

We consider canonical polynomials for initial value problem (1.1), for the cases $m=1,2, \ldots, s=1,2, \ldots$ and then generalize $Q_{n}(x) \forall m$ and $s$.

Case $m=1, s=1$ : $\operatorname{From}(1.1)$, we have

$$
\begin{gathered}
L y(x) \equiv\left(P_{0,0}+P_{0,1} x\right) y(x)+\left(P_{1,0}+P_{1,1} x+P_{1,2} x^{2}\right) y^{\prime}(x)=\sum_{r=0}^{\sigma} f_{r} x^{r} \\
\Rightarrow L x^{r}=\left(P_{0,0}+r P_{1,2}\right) x^{r+1}+\left(P_{0,0}+r P_{1,1}\right) x^{r}+r P_{1,0} x^{r-1}
\end{gathered}
$$

Since $L Q_{r}(x)=x^{r}$, we have

$$
Q_{r+1}(x)=\frac{x^{r}-r P_{1,0} Q_{r-1}(x)-\left(P_{0,0}+r P_{1,1}\right) Q_{r}(x)}{P_{0,1}+r P_{1,2}}, \quad r \geq 0 .
$$

Case $m=1, s=2$ :

$$
\begin{aligned}
& \left(P_{0,0}+P_{0,1} x+P_{0,2} x^{2}\right) y(x)+\left(P_{1,0}+P_{1,1} x+P_{1,2} x^{2}+P_{1,3} x^{3}\right) y^{\prime}(x)=\sum_{r=0}^{\sigma} f_{r} x^{r} \\
& \Rightarrow Q_{r+2}(x)=\frac{x^{r}-r P_{1,0} Q_{r-1}(x)-\left(P_{0,0}+r P_{1,1}\right) Q_{r}(x)-\left(P_{0,1}+r P_{1,2}\right) Q_{r+1}(x)}{P_{0,2}+r P_{1,3}} .
\end{aligned}
$$

Case $m=1, s=3$ : We have

$$
\begin{aligned}
& Q_{r+3}(x)= \\
& \frac{x^{r}-r P_{1,0} Q_{r-1}(x)-\left(P_{0,0}+r P_{1,1}\right) Q_{r}(x)-\left(P_{0,1}+r P_{1,2}\right) Q_{r+1}(x)-\left(P_{0,2}+r P_{1,3}\right) Q_{r+2}(x)}{P_{0,2}+r P_{1,4}} .
\end{aligned}
$$

Generally, for case $m=1, s=s$ we have

$$
\begin{aligned}
Q_{n}(x)=\frac{x^{n-s}-\left[\sum_{j=0}^{s}\left(P_{0, j-1}+(n-s) P_{1, j}\right) Q_{n-s+j-1}(x)\right]}{P_{0, s}+(n-s) P_{1, s+1}}, & \\
n=r+s, r \geq 0, &
\end{aligned}
$$

where $P_{0,-1}=0$ and $P_{0, s}+(n-s) P_{1, s+1} \neq 0$.

Case $m=2, s=1$ : From (1.1), we have 


$$
\begin{aligned}
\left(P_{2,0}+P_{2,1} x+P_{2,2} x^{2}+P_{2,3} x^{3}\right) y^{\prime \prime}(x)+ & \left(P_{1,0}+P_{1,1} x+P_{1,2} x^{2}\right) y^{\prime}(x) \\
& +\left(P_{0,0}+P_{0,1} x\right) y(x)=\sum_{r=0}^{\sigma} f_{r} x^{r},
\end{aligned}
$$

$Q_{r+1}(x)=$

$\frac{x^{r}-\left[\left(P_{0,0}+r P_{1,1}+r(r-1) P_{2,2}\right) Q_{r}(x)+\left(r P_{1,0}+r(r-1) P_{2,1}\right) Q_{r-1}(x)+r(r-1) P_{2,0} Q_{r-2}(x)\right]}{P_{0,1}+r P_{1,2}+r(r-1) P_{2,3}}$.

Case $m=2, s=2$ :

For this case we obtained

$$
\begin{aligned}
Q_{r+2}(x)= & \frac{1}{P_{0,2}+r P_{1,3}+r(r-1) P_{2,4}}\left(x^{r}-\left[\left(r(r-1) P_{2,3}+r P_{1,2}+P_{0,1}\right) Q_{r+1}(x)\right.\right. \\
& +\left(P_{0,0}+r P_{1,1}+r(r-1) P_{2,2}\right) Q_{r}(x) \\
& \left.\left.\left.+\left(r P_{1,0}+r(r-1) P_{2,1}\right) Q_{r-1}(x)+r(r-1) P_{2,0}\right) Q_{r-2}(x)\right]\right) .
\end{aligned}
$$

Generally, for case $m=2, s=s$ We have

$$
\begin{array}{r}
Q_{n}(x)=\frac{x^{n-s}-\left[\sum_{j=0}^{s}\left(P_{0, j-1}+r P_{1, j}+r(r-1) P_{2, j+1}\right) Q_{r+j-1}(x)\right]}{P_{0, s}+r P_{1, s+1}+r(r-1) P_{2, s+2}} \\
n=r+s, r \geq 0
\end{array}
$$

where $P_{0,-1}=0$ and $P_{0, s}+r P_{1, s+1}+r(r-1) P_{2, s+2} \neq 0$.

Case $m=m, s=s$ : Continuing the process above, then derived the general canonical polynomial for both overdetermined and non-overdetermined ordinary differential equations (see Yisa [14]).

$$
\begin{aligned}
& Q_{n}(x)= \\
& \frac{1}{\sum_{k=0}^{m} k !\left(\begin{array}{c}
n-s \\
k
\end{array}\right) P_{k, k+s}}\left\{x^{n-s}-\left[\sum_{k=1}^{m}\left(\sum_{j=k}^{m} j !\left(\begin{array}{c}
n-s \\
j
\end{array}\right) P_{j, j-k}\right)^{Q_{n-s-k}(x)}\right.\right. \\
& \left.\left.+\sum_{k=0}^{s-1}\left(\sum_{j=0}^{m} j !\left(\begin{array}{c}
n-s \\
j
\end{array}\right) P_{j, j+k}\right)^{Q_{n-s+k}(x)}\right\rfloor\right\} .
\end{aligned}
$$

Theorem 1. (see Yisa [14]) If

$$
L y(x) \equiv \sum_{r=0}^{m}\left(\sum_{k=0}^{N_{r}} P_{r k} x^{k}\right) y^{r}(x)=\sum_{r=0}^{\sigma} f_{r} x^{r}=f(x)
$$


where $P_{k}(x)$ is a $k$-th degree polynomials, $y^{r}(x)$ is the $r$-th derivative of $y(x)$ and $f(x)$ is a polynomial of degree $\sigma$,is an $m$-th order ordinary differential equation, then the canonical polynomial associated with its differential operator

$$
L=\sum_{r=0}^{m}\left(\sum_{k=0}^{N_{r}} P_{r k} x^{k}\right) \frac{d^{r}}{d x^{r}}
$$

is

$$
\begin{aligned}
& Q_{n}(x)= \\
& \frac{1}{\sum_{k=0}^{m} k !\left(\begin{array}{c}
n-s \\
k
\end{array}\right) P_{k, k+s}}\left\{x^{n-s}-\left[\sum_{k=1}^{m}\left(\sum_{j=k}^{m} j !\left(\begin{array}{c}
n-s \\
j
\end{array}\right) P_{j, j-k}\right)^{Q_{n-s-k}(x)}\right.\right. \\
& \left.\left.+\sum_{k=0}^{s-1}\left(\sum_{j=0}^{m} j !\left(\begin{array}{c}
n-s \\
j
\end{array}\right) P_{j, j+k}\right)^{Q_{n-s+k}(x)}\right\rfloor\right\} \text {. }
\end{aligned}
$$

\section{Derivation of Recursive Formulation of the Tau Approximant}

In this section, the tau approximant for the recursive form using the generalized canonical polynomial is considered for varying orders and degrees. From the perturbed equation(1.2)

$$
\begin{aligned}
& L y_{n}(x)=\sum_{r=0}^{\sigma} f_{r} x^{r}+H_{n}(x), \\
& L^{*} y_{n}\left(x_{r k}\right)=\alpha_{k}, k=1(1) m,
\end{aligned}
$$

where

$$
H_{n}(x)=\sum_{i=0}^{m+s-1} \tau_{i+1} T_{n-m+i+1}(x)=\sum_{i=0}^{m+s-1} \tau_{i+1} \sum_{r=0}^{n-m+i+1} C_{r}^{(n-m+i+1)} x^{r}
$$

is the perturbation term.

Case $m=1, s=1$ : We have

$$
L y_{n}(x)=\sum_{r=0}^{\sigma} f_{r} x^{r}+\sum_{i=0}^{1} \tau_{i+1} \sum_{r=0}^{n+i} C_{r}^{(n+i)} x^{r}
$$




$$
\begin{aligned}
& =\sum_{r=0}^{\sigma} f_{r} L Q_{r}(x)+\sum_{i=0}^{1} \tau_{i+1} \sum_{r=0}^{n+i} C_{r}^{(n+i)} L Q_{r}(x) \\
& =L\left\{\sum_{r=0}^{\sigma} f_{r} Q_{r}(x)+\sum_{i=0}^{1} \tau_{i+1} \sum_{r=0}^{n+i} C_{r}^{(n+i)} Q_{r}(x)\right\} \\
\Rightarrow y_{n}(x) & =\sum_{r=0}^{\sigma} f_{r} Q_{r}(x)+\tau_{1} \sum_{r=0}^{n} C_{r}^{(n)} Q_{r}(x)+\tau_{2} \sum_{r=0}^{n+1} C_{r}^{(n+1)} Q_{r}(x) .
\end{aligned}
$$

Now, inserting (2.4); expand the resulting equation and equate the coefficient of undetermined canonical polynomial (i.e $Q_{0}(x)$ ) to zero, to obtain

$$
\tau_{1} \sum_{r=0}^{n} C_{r}^{(n)} P_{r}+\tau_{2} \sum_{r=0}^{n+1} C_{r}^{(n+1)} P_{r}+\sum_{r=0}^{\sigma} f_{r} P_{r}=0 .
$$

Take $P_{0}=1$. Equation (3.1) is the coefficient of undetermined canonical polynomial (i.e $Q_{0}(x)$ ) and for ease generalization we assume $Q_{0}(x)=1$, to obtain

$$
y_{n}(x)=\sum_{r=1}^{\sigma} f_{r} q_{r}(x)+\sum_{i=0}^{1} \tau_{i+1} \sum_{r=1}^{n+i} C_{r}^{(n+i)} q_{r}(x),
$$

where $q_{n}=Q_{n}(x)-P_{n}$ and

$$
P_{n}=\frac{-P_{0,0} P_{n-1}-(n-1) P_{1,0} P_{n-2}-(n-1) P_{1,1} P_{n-1}}{P_{0,1}+(n-1) P_{1,2}} .
$$

Case $m=1, s=2$ : From (1.2), we have

$$
\begin{aligned}
y_{n}(x)=\sum_{r=0}^{\sigma} f_{r} Q_{r}(x)+\tau_{1} \sum_{r=0}^{n} C_{r}^{(n)} Q_{r}(x)+\tau_{2} \sum_{r=0}^{n+1} C_{r}^{(n+1)} Q_{r}(x) & \\
& +\tau_{3} \sum_{r=0}^{n+2} C_{r}^{(n+2)} Q_{r}(x) .
\end{aligned}
$$

Again, insert (2.4); expand the resulting equation and equate the coefficient of undetermined canonical polynomials (i.e $Q_{0}(x), Q_{1}(x)$ ) to zero, to obtain

$$
\tau_{1} \sum_{r=0}^{n} C_{r}^{(n)} P_{r}+\tau_{2} \sum_{r=0}^{n+1} C_{r}^{(n+1)} P_{r}+\tau_{3} \sum_{r=0}^{n+2} C_{r}^{(n+2)} P_{r}+\sum_{r=0}^{\sigma} f_{r} P_{r}=0 .
$$


Take $P_{0}=1, P_{1}=0$ for coefficient of $Q_{0}(x)$, for coefficient of $Q_{1}(x)$ take $P_{0}=0$, $P_{1}=1$ and for $y_{n}(x)$ we assume $Q_{0}(x)=Q_{1}(x)=1$, and take $P_{0}=P_{1}=1$, to obtain

$$
y_{n}(x)=\sum_{r=2}^{\sigma} f_{r} q_{r}(x)+\sum_{i=0}^{2} \tau_{i+1} \sum_{r=2}^{n+i} C_{r}^{(n+i)} q_{r}(x),
$$

where

$$
\begin{aligned}
& P_{n}= \\
& \frac{-(n-2) P_{1,0} P_{n-3}-\left(P_{0,0}+(n-2) P_{1,1}\right) P_{n-2}-\left(P_{0,1}+(n-2) P_{1,2}\right) P_{n-1}}{P_{0,2}+(n-2) P_{1,3}},
\end{aligned}
$$

and $q_{n}=Q_{n}(x)-P_{n}$.

Case $m=2, s=1$ : Insert (2.4) in (1.2); expand the resulting equation and equate the coefficient of undetermined canonical polynomial (i.e $Q_{0}(x)$ ) to zero, to obtain

$$
\tau_{1} \sum_{r=0}^{n-1} C_{r}^{(n-1)} P_{r}+\tau_{2} \sum_{r=0}^{n} C_{r}^{(n)} P_{r}+\tau_{3} \sum_{r=0}^{n+1} C_{r}^{(n+1)} P_{r}+\sum_{r=0}^{\sigma} f_{r} P_{r}=0 .
$$

Take $P_{0}=1$ for the coefficient of $Q_{0}(x)$, and for $y_{n}(x)$ we assume $Q_{0}(x)=P_{0}=$ 1 , then we obtain the solution

$$
y_{n}(x)=\sum_{r=1}^{\sigma} f_{r} q_{r}(x)+\sum_{i=0}^{2} \tau_{i+1} \sum_{r=1}^{n-1+i} C_{r}^{(n-1+i)} q_{r}(x)
$$

and

$$
y_{n}^{\prime}(x)=\sum_{r=1}^{\sigma} f_{r} Q_{r}^{\prime}(x)+\sum_{i=0}^{2} \tau_{i+1} \sum_{r=1}^{n+i} C_{r}^{(n-1+i)} Q_{r}^{\prime}(x) .
$$

Case $m=2, s=2$ : Insert (2.4) in (1.2); expand the resulting equation and equate the coefficient of undetermined canonical polynomials (i.e $Q_{0}(x)$ and $\left.Q_{1}(x)\right)$ to zero to obtain

$$
\begin{aligned}
\tau_{1} \sum_{r=0}^{n-1} C_{r}^{(n-1)} P_{r}+\tau_{2} \sum_{r=0}^{n} C_{r}^{(n)} P_{r}+\tau_{3} \sum_{r=0}^{n+1} C_{r}^{(n+1)} P_{r}+\tau_{4} \sum_{r=0}^{n} C_{r}^{(n+2)} P_{r} & \\
& +\sum_{r=0}^{\sigma} f_{r} P_{r}=0
\end{aligned}
$$


for the coefficient of $Q_{0}(x)$, take $P_{0}=1$ and $P_{1}=0$, and for the coefficient of $Q_{1}(x)$, take $P_{1}=1, P_{0}=0$ and for $y_{n}(x)$ we assume $Q_{0}(x)=Q_{1}(x)=P_{0}=$ $P_{1}=1$, then we obtain the solution

$$
y_{n}(x)=\sum_{r=2}^{\sigma} f_{r} q_{r}(x)+\sum_{i=0}^{3} \tau_{i+1} \sum_{r=1}^{n-1+i} C_{r}^{(n-1+i)} q_{r}(x)
$$

and

$$
y_{n}^{\prime}(x)=\sum_{r=2}^{\sigma} f_{r} Q_{r}^{\prime}(x)+\sum_{i=0}^{3} \tau_{i+1} \sum_{r=1}^{n+i} C_{r}^{(n-1+i)} Q_{r}^{\prime}(x) .
$$

Observing the trend in the tau approximant of the first and second order ordinary differential equations presented above, we derived the general formula

$$
y_{n}(x)=\sum_{r=s}^{\sigma} f_{r} q_{r}(x)+\sum_{i=0}^{m+s-1} \tau_{i+1} \sum_{r=s}^{n-m+i+1} C_{r}^{(n-m+i+1)} q_{r}(x) .
$$

Assume $Q_{r}(x)=P_{r}=1, r=0(1)(s-1)$

$$
\begin{gathered}
y_{n}^{\lambda}(x)=\sum_{r=s}^{\sigma} f_{r} Q_{r}^{\lambda}(x)+\sum_{i=0}^{m+s-1} \tau_{i+1} \sum_{r=s}^{n-m+i+1} C_{r}^{(n-m+i+1)} Q_{r}^{\lambda}(x)=\alpha_{\lambda}, \\
\lambda=0(1)(m-1), \\
\sum_{i=0}^{m+s-1} \tau_{i+1} \sum_{r=0}^{n-m+i+1} C_{r}^{(n-m+i+1)} P_{r}+\sum_{r=0}^{\sigma} f_{r} P_{r}=0,
\end{gathered}
$$

where $q_{n}(x)=Q_{n}(x)-P_{n}$ and equation (3.11) is the coefficient of undetermined canonical polynomials.

$$
\begin{aligned}
P_{n}=\frac{-1}{\sum_{k=0}^{m} k !\left(\begin{array}{c}
n-s \\
k
\end{array}\right) P_{k, k+s}}\{ & \left\{\sum_{k=1}^{m}\left(\sum_{j=k}^{m} j !\left(\begin{array}{c}
n-s \\
j
\end{array}\right) P_{j, j-k}\right)^{P_{n-s-k}}\right. \\
& \left.+\sum_{k=0}^{s-1}\left(\sum_{j=0}^{m} j !\left(\begin{array}{c}
n-s \\
j
\end{array}\right) P_{j, j+k}\right)^{P_{n-s+k}}\right\} .
\end{aligned}
$$

Take $P_{r}=1$, when equating the coefficient of $Q_{r}(x)$ to zero, otherwise $P_{r}=0$, $r=0(1)(s-1)$.

The formula also valid for non-overdetermined differential equation, by replacing $s=0$ in (2.4), $P_{r}=0, \forall r$ in (3.9) and neglect (3.11) and (3.12). 


\section{Numerical Experiments}

In this section we present some numerical experiments to illustrate the numerical results presented in this paper. The exact errors are defined as $\varepsilon_{n}=a \leq x \leq b$ $\left\{\mid y\left(x_{k}-y_{n}\left(x_{k} \mid\right\}\right.\right.$, where $\left\{x_{k}\right\}=(0.01 k), k=0(1) 100$.

\section{Problem 1.}

$$
L y(x)=y^{\prime}(x)+2 x y(x)=4 x, y(0)=3,
$$

with analytical solution $y(x)=2+\exp \left(-x^{2}\right)$.

Comparing with (1.2), $P_{0,0}=0, P_{0,1}=2, P_{1,0}=1, P_{1,1}=P_{1,2}=0, f_{0}=0$, $f_{1}=4, \sigma=1, m=1, s=1$.

For degree 5 (i.e $n=5$ ), applying equations (3.9) and (3.11), we have

$$
y_{5}(x)=3+\frac{164 x}{3360543}-\frac{3356272 x^{2}}{3360543}-\frac{29952 x^{3}}{1120181}+\frac{2100992 x^{4}}{3360543}-\frac{779264 x^{5}}{3360543} .
$$

For degree 6 (i.e $n=6$ ), we have

$$
\begin{array}{r}
y_{6}(x)=3-\frac{35308 x}{160301129}-\frac{159018016 x^{2}}{160301129}-\frac{10087168 x^{3}}{160301129}+\frac{112491776 x^{4}}{160301129} \\
-\frac{48468992 x^{5}}{160301129}+\frac{3788800 x^{6}}{160301129} .
\end{array}
$$

For degree 7 (i.e $n=7$ ), we have

$$
\begin{aligned}
y_{7}(x)=3-\frac{560 x}{56926267}-\frac{56902112 x^{2}}{56926267}-\frac{210560 x^{3}}{56926267}+\frac{29105920 x^{4}}{56926267} \\
-\frac{403456 x^{5}}{56926267}-\frac{11161600 x^{6}}{56926267}+\frac{3588096 x^{7}}{56926267} .
\end{aligned}
$$

\section{Problem 2.}

$$
L y(x)=y^{\prime}(x)-x^{2} y(x)=0, \quad y(0)=1,
$$

with analytical solution $y(x)=\exp \left(\frac{1}{3} x^{3}\right)$.

For degree 5, 6 and 7 approximate solution, we have

$$
\begin{array}{r}
y_{5}(x)=1+\frac{42887122 x}{26442910625}-\frac{1012427712 x^{2}}{26442910625}+\frac{13794322304 x^{3}}{26442910625}-\frac{9264926976 x^{4}}{26442910625} \\
+\frac{6901011968 x^{5}}{26442910625}
\end{array}
$$




$$
\begin{array}{r}
y_{6}(x)=1-\frac{5102898720 x}{20423863454407}+\frac{172910611296 x^{2}}{20423863454407}+\frac{795917236736 x^{3}}{2917694779201} \\
+\frac{3494579851008 x^{4}}{20423863454407}-\frac{4385551601664 x^{5}}{20423863454407}+\frac{3231878008832 x^{6}}{20423863454407}, \\
y_{7}(x)=1+\frac{54229310046 x}{1148232742907190}-\frac{2553117942456 x^{2}}{1148232742907190}+\frac{81717617906288 x^{3}}{229646548581439} \\
-\frac{108270560916864 x^{4}}{1148232742907190}+\frac{44581151086848 x^{5}}{229646548581439}-\frac{165543248190464 x^{6}}{1148232742907190} \\
+\frac{99073874491392 x^{7}}{1148232742907190} .
\end{array}
$$

respectively.

\section{Problem 3.}

$$
L y(x)=y^{\prime \prime}(x)-2\left(1+2 x^{2}\right) y(x)=0, \quad y(0)=1, \quad y^{\prime}(0)=0,
$$

with analytical solution $y(x)=\exp \left(x^{2}\right)$.

For degree 5, 6 and 7 approximate solution, we have

$$
\begin{aligned}
y_{5}(x)=1+\frac{19855060080 x^{2}}{21441580663}+\frac{15314739712 x^{3}}{21441580663}-\frac{22091646208 x^{4}}{21441580663} \\
+\frac{23992840192 x^{5}}{21441580663} .
\end{aligned}
$$

$$
\begin{aligned}
y_{6}(x)=1+\frac{14339349817120 x^{2}}{14137226504329} & -\frac{3116501231104 x^{3}}{14137226504329}+\frac{54597872159488 x^{4}}{42411679512987} \\
& -\frac{46782632538112 x^{5}}{42411679512987}+\frac{31339103866880 x^{6}}{42411679512987} .
\end{aligned}
$$

$$
\begin{aligned}
& y_{7}(x)=1+ \frac{28873617687738500 x^{2}}{28953089655559100}+\frac{1809682067903230 x^{3}}{28953089655559100} \\
&+\frac{4638442954437370 x^{4}}{28953089655559100}+\frac{22820567800864700 x^{5}}{28953089655559100} \\
&-\frac{20305651810889700 x^{6}}{28953089655559100}+\frac{11916952869830600 x^{7}}{28953089655559100},
\end{aligned}
$$

respectively.

For experiment 4 and 5, we consider non-overdetermined type. 


\section{Problem 4.}

$$
L y(x)=2(1+x) y^{\prime}(x)+y(x)=0, \quad y(0)=1, \quad 0 \leq x \leq 1,
$$

with analytical solution $y(x)=(1+x)^{\frac{-1}{2}}, P_{0,0}=1, P_{1,0}=2, P_{1,1}=2, m=1$, $f_{0}=0$.

For degree 5, 6 and 7 approximate solution, we have

$$
\begin{array}{r}
y_{5}(x)=1-\frac{169546 x}{339323}+\frac{124272 x^{2}}{339323}-\frac{88160 x^{3}}{339323}+\frac{44800 x^{4}}{339323}-\frac{10752 x^{5}}{339323}, \\
y_{6}(x)=1-\frac{59392456 x}{118799927}+\frac{44274072 x^{2}}{118799927}-\frac{34792960 x^{3}}{118799927}+\frac{23717120 x^{4}}{118799927}-\frac{645120 x^{5}}{6988231} \\
+\frac{2365440 x^{6}}{118799927} \\
y_{7}(x)=1-\frac{9239102 x}{18478633}+\frac{6918816 x^{2}}{18478633}-\frac{5653568 x^{3}}{18478633}+\frac{4442368 x^{4}}{18478633}-\frac{14224896 x^{5}}{92393165} \\
+\frac{5992448 x^{6}}{92393165}-\frac{1171456 x^{7}}{92393165} .
\end{array}
$$

\section{Problem 5.}

$$
y^{\prime \prime}(x)-(1-x) y^{\prime}(x)+y(x)=0, \quad y(0)=1, \quad y^{\prime}(0)=1, \quad 0 \leq x \leq 1,
$$

with analytical solution $\exp \left(x-\frac{x^{2}}{2}\right)$.

For degree 5, 6 and 7 approximate solution, we have

$$
\begin{gathered}
y_{5}(x)=1+x+\frac{2168 x^{2}}{3272391}-\frac{3315848 x^{3}}{9817173}-\frac{815488 x^{4}}{9817173}+\frac{675584 x^{5}}{9817173}, \\
y_{6}(x)=1+x-\frac{230528 x^{2}}{515794089}-\frac{504319192 x^{3}}{1547382267}-\frac{173802752 x^{4}}{1547382267}+\frac{149575936 x^{5}}{1547382267} \\
-\frac{51200 x^{6}}{5546173}, \\
y_{7}(x)=1+x-\frac{57032 x^{2}}{42919912639}-\frac{42921645520 x^{3}}{128759737917}-\frac{10673371904 x^{4}}{128759737917} \\
+\frac{6122414848 x^{5}}{128759737917}+\frac{1202865152 x^{6}}{42919912639}-\frac{455455744 x^{7}}{42919912639} .
\end{gathered}
$$

Below are the maximum errors obtain for each experiment and degree. 


\begin{tabular}{|c|c|c|c|}
\hline Experiment & $\varepsilon_{5}$ & $\varepsilon_{6}$ & $\varepsilon_{7}$ \\
\hline Problem 1 & $3.57 \times 10^{-5}$ & $1.97 \times 10^{-5}$ & $1.10 \times 10^{-6}$ \\
\hline Problem 2 & $2.07 \times 10^{-4}$ & $2.96 \times 10^{-5}$ & $4.50 \times 10^{-6}$ \\
\hline Problem 3 & $1.06 \times 10^{-2}$ & $1.23 \times 10^{-3}$ & $1.40 \times 10^{-4}$ \\
\hline Problem 4 & $3.08 \times 10^{-5}$ & $4.24 \times 10^{-6}$ & $6.64 \times 10^{-7}$ \\
\hline Problem 5 & $9.42 \times 10^{-5}$ & $2.62 \times 10^{-5}$ & $1.81 \times 10^{-7}$ \\
\hline
\end{tabular}

Table 1: Errors of Experiments 1-5

\section{Conclusion}

The generalized form of the recursive formulation of the tau method for initial value problems in ordinary differential equations for both overdetermined and non-overdetermined types has been presented. Evidently, the overdetermined type is more cumbersome to derive than for the case of non-overdetermined type.

This study will facilitate an automation of this variant of the tau method towards a software development of the technique.

The derived scheme has been implemented on some selected test problems. Numerical evidences therefrom confirm its accuracy and effectiveness.

\section{References}

[1] R.B. Adeniyi, A.I. Ma'ali, An error estimation of the tau method for some ordinary differential equations, IOSR J. Math., 2, No. 1 (2012), 32-40.

[2] R.B. Adeniyi, A.I.M. Aliyu, On the tau method for a class of nonoverdetermined second order differential equations, Research J. of Appl. Sci., 3, No. 6 (2008), 438-446.

[3] R.B. Adeniyi, K. Issa, An analogue of the tau method for ordinary differential equations, Global J. Maths. and Stat., 2 (2010), 161-170.

[4] R.B. Adeniyi, E.O. Edungbola, On the tau method for certain overdetermined first order differential equations, J. Nig. Ass. Mathematical Physics Soc., 12 (2008), 399-408.

[5] R.B. Adeniyi, E.O. Edungbola, On the recursive formulation of the tau method for class of overdetermined first order equations, Abacus J. Math. Assoc. Nig., 34, No. 2B (2007), 249-261. 
[6] R.B. Adeniyi, B.M. Yisa, On the variants of the tau methods for solutions of IVPs in first ordinary differential systems, Kenya J. of Sci., Series A, 15, No. 1 (2012), 12-26.

[7] L. Fox, Numerical Solution of Ordinary and Partial Differential Equations, Pergamen Press Oxford (1968).

[8] M.K. El-Daou, A tau method with the perturbed boundary conditions for certain ODEs, Numerical Algorithms, 38 (2005), 31-45.

[9] M.K. El-Daou, E.L. Ortiz, Weight subspaces of the tau method and orthogonal collocation, J. Math. Anal. Appl., 326 (2007), 622-631.

[10] L. Fox, I.B. Parker, Chebyshev Polynomials in Numerical Analysis, University Press Oxford (1968).

[11] C. Lanczos, Applied Analysis, New Jersey (1956).

[12] C. Lanczos, Trigonometric interpolation of empirical and analytic functions, J. Math. and Physics, 17 (1938), 123-199.

[13] E.L. Ortiz, The tau method, SIMAJ. Numer. Anal., 6 (1969), 480-492.

[14] B.M. Yisa, R.B. Adeniyi, Generalization of canonical polynomials for overdetermined $m$ - th order ordinary differential equations (ODEs), IJERT Journal, 1, No. 6 (2012), 1-15. 
